\title{
Reactor antineutrino monitoring with a plastic scintillator array as a new safeguards method
}

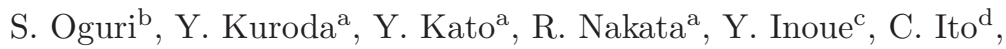 \\ M. Minowa ${ }^{\mathrm{a}, *}$ \\ ${ }^{a}$ Department of Physics, School of Science, the University of Tokyo, 7-3-1, Hongo, \\ Bunkyo-ku, Tokyo 133-0033, Japan \\ ${ }^{b}$ Institute of Particle and Nuclear Studies, High Energy Accelerator Research Organization \\ (KEK), 1-1, Oho, Tsukuba, Ibaraki 305-0801, Japan \\ ${ }^{c}$ International Center for Elementary Particle Physics, the University of Tokyo, 7-3-1, \\ Hongo, Bunkyo-ku, Tokyo 133-0033, Japan \\ ${ }^{d}$ Oarai Research and Development Center, Japan Atomic Energy Agency, 4002, Naritacho, \\ Oarai-machi, Higashiibaraki-gun, Ibaraki 311-1393, Japan
}

\begin{abstract}
We developed a segmented reactor-antineutrino detector made of plastic scintillators for application as a tool in nuclear safeguards inspection and performed mostly unmanned field operations at a commercial power plant reactor. At a position outside the reactor building, we measured the difference in reactor antineutrino flux above the ground when the reactor was active and inactive.

Keywords: Reactor, Safeguards, Neutrino, Antineutrino
\end{abstract}

\section{Introduction}

A half a century ago, neutrinos were first discovered at a nuclear reactor plant by Fred Reines, Clyde Cowan and their colleagues 11]. Indeed, nuclear reactors are the most intense man-controlled sources of neutrinos. A total flux of $2 \times 10^{20}$ antineutrinos/s is emitted by a $1-\mathrm{GW}_{\text {th }}$ power plant $\left.2-4\right]$. In recent years, neutrino physics is studied intensively as a means to monitor reactor operations.

\footnotetext{
*Corresponding author

Email address: minowa@phys.s.u-tokyo.ac.jp (M. Minowa)
}

Preprint submitted to Nuclear Instruments and Methods in Physics Research AAugust 13, 2018 
International Atomic Energy Agency(IAEA) uses an extensive set of technical measures by which it independently verifies the correctness and completeness of declarations made by countries about their stores of nuclear material and activities. IAEA recommends [5] near-field antineutrino monitoring capabilities to provide operational status, thermal power, and fissile content of reactors to ensure the implementation of reactor safeguards. The merits of using antineutrinos physics are as follows:

- Non-intrusiveness

Because of their high penetration, antineutrinos can be detected outside reactor buildings.

- No other sources

Because comparable fluxes of antineutrinos are difficult to create without using reactors or accelerators, one can therefore obtain raw data of a reactor.

- Information of the isotopic content

By measurement of the antineutrino energy spectrum, one can determine not only the operational status and thermal power of a reactor, but also fissile content [6].

IAEA proposed the development of a compact detector within a standard 12meter ISO container (approximately 25,000 kg net load) and the aboveground deployment as medium term (5-8 year timeframe) goals [5]. The aboveground deployment is very important because of its non-intrusiveness. However, it is a challenge because of background noise induced by cosmic rays and as yet no group has succeeded in producing a working prototype, despite several endeavors among various groups [6 10] around the world.

Taking the above points into account, we proposed a segmented antineutrino detector, PANDA, an acronym for plastic anti-neutrino detector array [11]. Because of its segmented structure and its use of event topology information, PANDA has a strong background rejection capability. 
In the first stage of the PANDA project, we built and operated a small prototype detector called Lesser PANDA, at the Unit 3 reactor of the Hamaoka Nuclear Power Plant of the Chubu Electric Power Co., Inc. It consisted of 16 modules of plastic scintillators and a total target mass of $160 \mathrm{~kg}$. We had planned to measure the change in antineutrino flux during the startup of the Unit 3 reactor, but the reactor was not brought online because of the 2011 Tohoku earthquake off the Pacific Coast of Japan. We measured background data for two months there. The results were reported in [11].

In the next step of our project, we constructed a 360-kg prototype neutrino detector called PANDA36 as a tool to inspect and assess safeguards. Over a twomonth period we demonstrated its operation above ground at 36 meters away from the $3.4 \mathrm{GW}_{\text {th }}$ reactor core of Ohi Power Station of the Kansai Electric Power Co., Inc. The purpose of the experiment was two-fold: the detection of the change in antineutrino flux between online and off-line reactor periods and the analysis of background flux from above-ground measurements for feasibility assessment of project goals. In this paper, we report results of the experiment using the prototype detector PANDA36.

\section{The detector}

\subsection{Principle and features}

We detect antineutrinos via the inverse beta decay interaction on a proton in the plastic scintillator with the energy threshold of $1.8 \mathrm{MeV}$.

$$
\overline{\nu_{e}}+p \rightarrow e^{+}+n \text {. }
$$

The positron and the neutron which are produced by the inverse beta decay are detected independently. The positron deposits energy via ionization, and emits two gamma rays by annihilation:

$$
e^{+}+e^{-} \rightarrow 2 \gamma
$$

It is referred to as the prompt event in this paper hereafter. The neutron is thermalized in the plastic and captured some time later by gadolinium embedded 


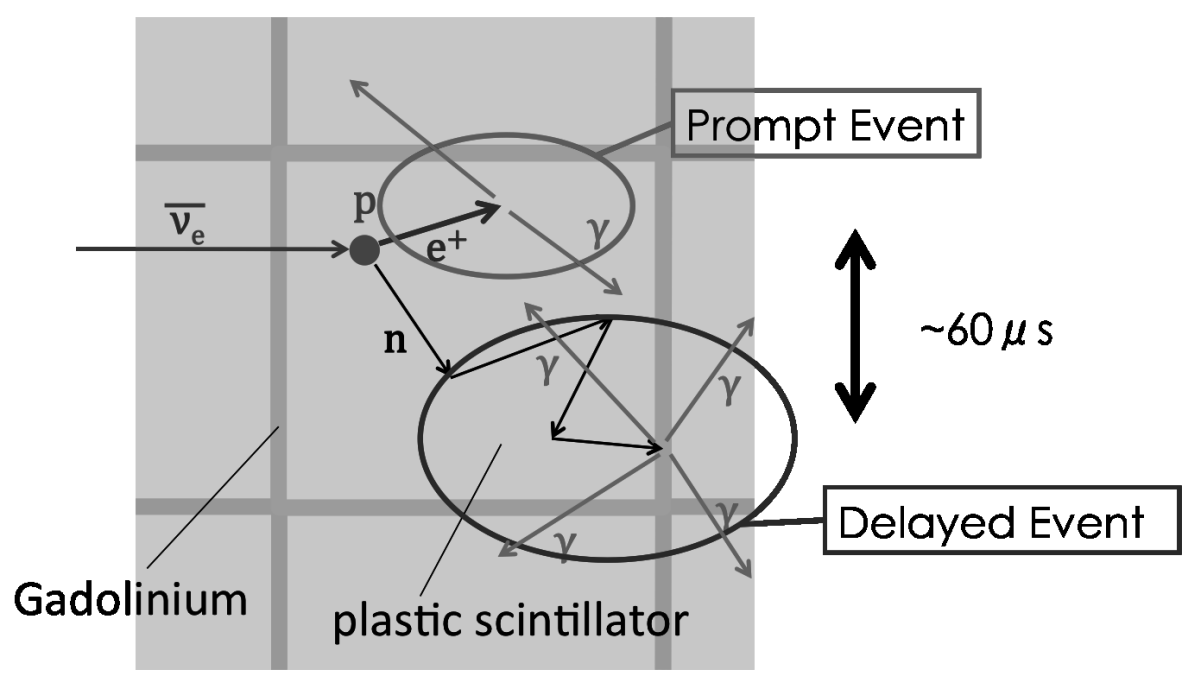

Figure 1: Principle of antineutrino detection with a cross-sectional view of the pillar modules

in between plastic scintillators, and a gamma ray cascade is produced with total energy of about $8 \mathrm{MeV}$ :

$$
\begin{aligned}
& n+{ }^{155} \mathrm{Gd} \rightarrow{ }^{156} \mathrm{Gd}^{*} \rightarrow{ }^{156} \mathrm{Gd}+\gamma^{\prime} \mathrm{s}, \\
& n+{ }^{157} \mathrm{Gd} \rightarrow{ }^{158} \mathrm{Gd}^{*} \rightarrow{ }^{158} \mathrm{Gd}+\gamma^{\prime} \mathrm{s} .
\end{aligned}
$$

It is referred to as the delayed event. The prompt and the delayed events are detected in delayed coincidence. The principle of detection is illustrated in Fig. 1 .

Our detector has four original features as follows.

- Mobility

The target mass of PANDA36 detector is $360 \mathrm{~kg}$. It is small as a neutrino detector. In addition, our detector is loaded into a van, and can operate in the van.

- Solid state

There are two merits of the solid state scintillator. The first is the easiness of transportation compared to liquid scintillator. Our detector is fully prepared in the van for the measurement and can be carried to the reactor 
as it is. The second is the non-flammability. Oil-based liquid scintillator is flammable and flammable oil is prohibited in many cases to be brought to commercial reactor sites.

- Aboveground measurement

Sea-level operation of reactor neutrino detectors is one of the greatest issues for the safeguards application. The previous experiments to detect reactor neutrinos are conducted in underground sites $[6$, , 7]. In contrast, our detector is deployed just outside a reactor building. If we detect the reactor neutrino, PANDA project is to be the first successful aboveground experiment.

But it is a difficult challenge. Above ground, there are higher background resulted from cosmic rays. Especially, neutrons produced by cosmic muons are difficult to be discriminated from delayed events of inverse beta decays.

- Segmented detector

In order to operate the reactor monitor aboveground, a powerful background rejection technique is needed. Our detector is segmented and the energy deposit in each module is recorded. So, it becomes possible to use the event topology information to tag antineutrino events and to discriminate them from background.

Generally, liquid scintillators are used for the reactor neutrino experiment because they are easy to be doped with gadolinium. But the technique to create clear and colorless Gd doped plastic scintillator is less established. Our solution to the issue is to use the segmented pillar plastic scintillators which are wrapped in gadolinium coated sheets.

\subsection{PANDA36 detector}

The sketch of PANDA36 detector is shown in Fig. 2. The detector consists of 36 identical modules which are the same ones as were used in the Lesser PANDA

detector 11]. The modules are referred to as the PANDA modules. Schematic view of a PANDA module is shown Fig. 3. Each PANDA module has $10 \mathrm{~kg}$ of 


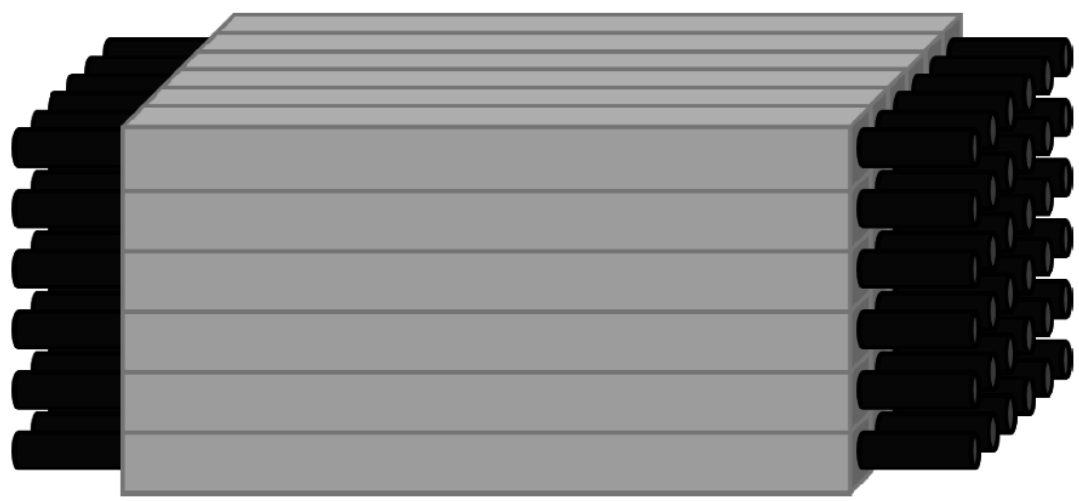

Figure 2: Schematic view of PANDA36 detector

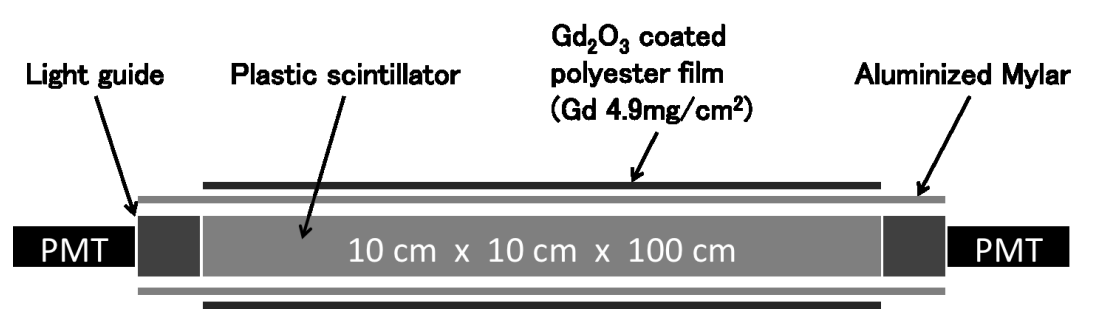

Figure 3: Schematic view of PANDA module

plastic scintillator (EJ-200, ELJEN Technology or RP-408, Rexon Technology) in it. Two $10 \mathrm{~cm} \times 10 \mathrm{~cm} \times 10 \mathrm{~cm}$ acrylic cubic light guides are glued to both ends of the plastic scintillator with optical cement (EJ-500, ELJEN Technology).

Two of 2-inch diameter PMTs(H6410, Hamamatsu) are glued to the light guides. The plastic scintillator and the light guides are wrapped in aluminized Mylar and gadolinium-oxide coated polyester sheet. The polyester sheet is obtained from Ask Sanshin Engineering Corp., Ltd. The sheet is made of $50 \mu \mathrm{m}$ thick polyester film sandwiched in two layers of $25-\mu$ m thick $\mathrm{Gd}_{2} \mathrm{O}_{3}$ coating. The sheet contains $4.9 \mathrm{mg} / \mathrm{cm}^{2}$ of gadolinium.

Schematic diagram of the DAQ system is shown in Fig. 4 The model number of each component which constructs the DAQ system is listed in Tab. 1 The 


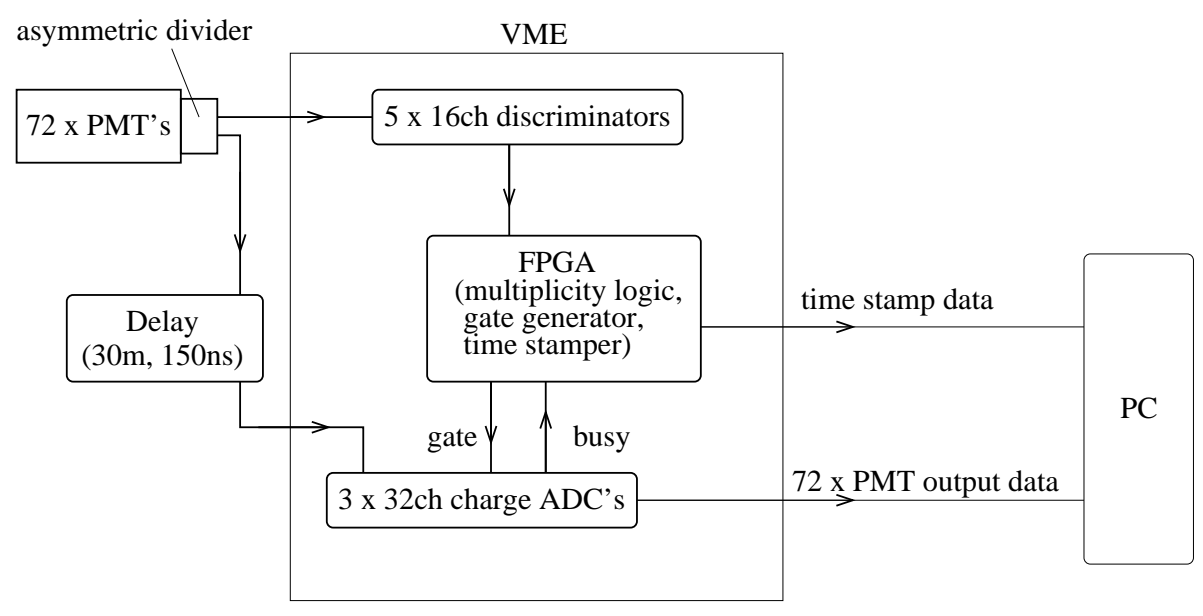

Figure 4: Schematic view of the DAQ system

Table 1: Table of model numbers of DAQ components

\begin{tabular}{|c|c|}
\hline component & model number \\
\hline \hline photomultiplier tube & Hamamatsu Photonics H6410 \\
\hline high voltage power supply & Matsusada Precison HARb-2N150 \\
\hline 16ch discriminator & CAEN V895 \\
\hline general purpose board (FPGA) & CAEN V1495 \\
& A395A, A395C \\
\hline 32ch charge ADC(QDC) & CAEN V792 \\
\hline
\end{tabular}




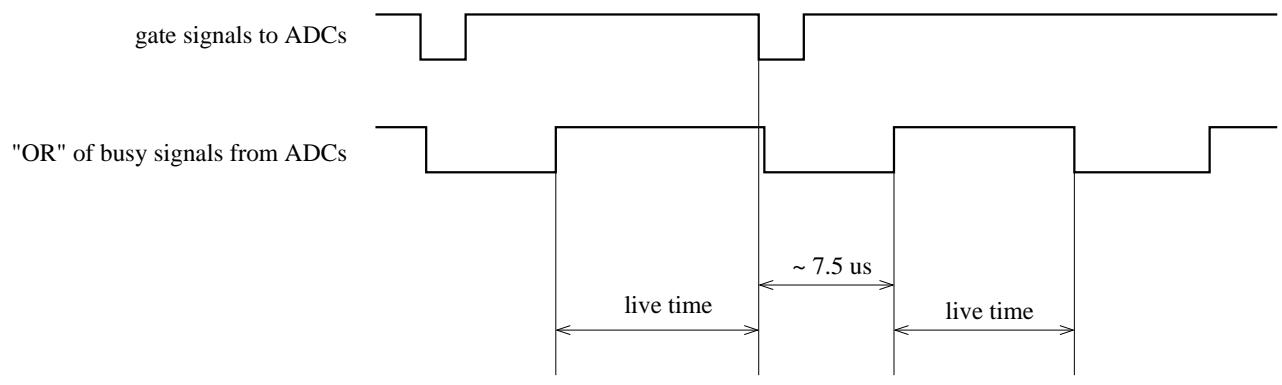

Figure 5: Timing diagram for live times

current pulses of PMTs are divided into two lines by passive signal dividers, where $15 \%$ of each current pulse enters a multievent charge ADC (QDC) through a $30 \mathrm{~m}$ delay cable(150 ns delay). The output of QDCs is recorded by a personal computer via VME bus. The rest of the current enters a discriminator and is then sent to a programmable FPGA board. The threshold of each discriminator is set to $150 \mathrm{keV}$ energy deposit equivalent at the far end from the corresponding PMTs.

The FPGA takes coincidence of two PMT signals of each PANDA module individually and gets 36 module-wise coincidence signals. The FPGA sends a gate signal of 400-ns width to the QDCs when there are at least 2 coincidence signals out of 16 ( 4 by 4 ) inner modules. We did not use 20 outer modules for the event trigger. At the same time, the FPGA records the time stamp of the leading edge of the QDC gate. The time stamps of both the leading and trailing edges of the busy signal from the QDCs are also recorded when its state has changed. The time stamp data are stored temporally in an internal FIFO of the FPGA and are read and recorded by the same PC via VME bus.

Data from the QDCs and the FPGA are combined based on the common event numbers which are embedded in the data. The event number is incremented by one on every event independently.

We measured live time of the DAQ using the time stamp data from FPGA. 
In many cases a busy signal is generated soon after a gate signal, and has a width of QDC conversion time of about $7.5 \mu \mathrm{s}$. The busy signals are also asserted without any relating gate signals when the QDCs are waiting for the data transfer. We took time intervals for the live time while neither the gate signal to the QDCs nor any of the busy signals of the QDCs were asserted. The timing diagram is shown in Fig. 囵

Energy calibrations with ${ }^{60} \mathrm{Co}$ gamma ray sources were carried out on 11th November 2011 at Hongo Campus of the University of Tokyo. First, energy calibration of each module was carried out using the Compton edge of ${ }^{60} \mathrm{Co}$ because the thickness of each plastic scintillator module is not enough for the total absorption.

In the next step, the total energy deposit for the detector as a whole was further calibrated using the total absorption peak of ${ }^{60} \mathrm{Co}$ by applying an overall normalization factor to all the modules since calibration by the Compton edge is prone to be affected by the uncertainty in the detector model.

The calibration source was inserted from the side into 1-cm-gap slits between the modules. Three calibration data at center and both ends were taken for each module.

There is also time variation of gains of the PMTs. We corrected the gains for each data set using the peak of through-going cosmic muons in the spectrum of the events in the data set by the minimum chi square method. The relativistic muons deposit energy of about $20 \mathrm{MeV}$ in $10 \mathrm{~cm}$ thickness of the plastic scintillator. The reference data were measured on 11th November 2011 at the same time as the calibration measurement.

PANDA36 was deployed at Ohi Power Station during the period from 18th November 2011 till 18th January 2012. The detector in the van was placed at a standoff of $35.9 \pm 0.1 \mathrm{~m}$ from the Ohi Unit 2 reactor core outside of the reactor building as is illustrated in Fig. 6. There were neither cosmic ray veto counters nor passive shields surrounding the detector. Ohi Unit 2 reactor was in operation at a thermal power of $3.4 \mathrm{GW}_{\mathrm{th}}$. We continued the measurement even after the reactor shutdown on 16th December to take background data at 
the same place for the rest of the time of about a month. In the measurement period, the other reactors(Unit 1,3, and 4) in Ohi Power Station were not in operation.

\section{Analysis}

By the delayed coincidence technique, two kinds of events are picked up by the data acquisition system. The first kind is referred to as the correlated event, in which a prompt event is correlated with a neutron which is captured certain time later by a Gd nucleus. The second kind is referred to as the uncorrelated event, which is the accidental coincidence of two independent events caused by natural backgrounds.

Besides antineutrino inverse beta decay interactions, fast neutrons produced by cosmic ray interactions can form the correlated events. A fast neutron scatters off a proton and gives a prompt energy deposition, then is captured by Gd with a characteristic time delay.

There is also another type of correlated background which consists of two cosmogenic fast neutrons produced at the same time by a muon spallation. We call it double-neutron correlated background. If both the neutrons are captured by Gd's and the earlier capture cannot be discriminated from the positron signal, they could also cause a correlated background. This kind of correlated background is, however, efficiently eliminated using individual information of the detector segments as is explained below.

Therefore, elimination of the fast neutron correlated background is the key issue to the detection of relatively small number of antineutrino events in overwhelming cosmic ray exposure environment above the ground.

We applied the selection cuts shown in Tab. 2 to the recorded events to pick up antineutrino events and to reduce background.

There are two sets of selection criteria, "selection 1" and "selection 2". First, we picked up the antineutrino-like events by selection 1. But the selected events also contain a certain fraction of fast neutron background because those back- 


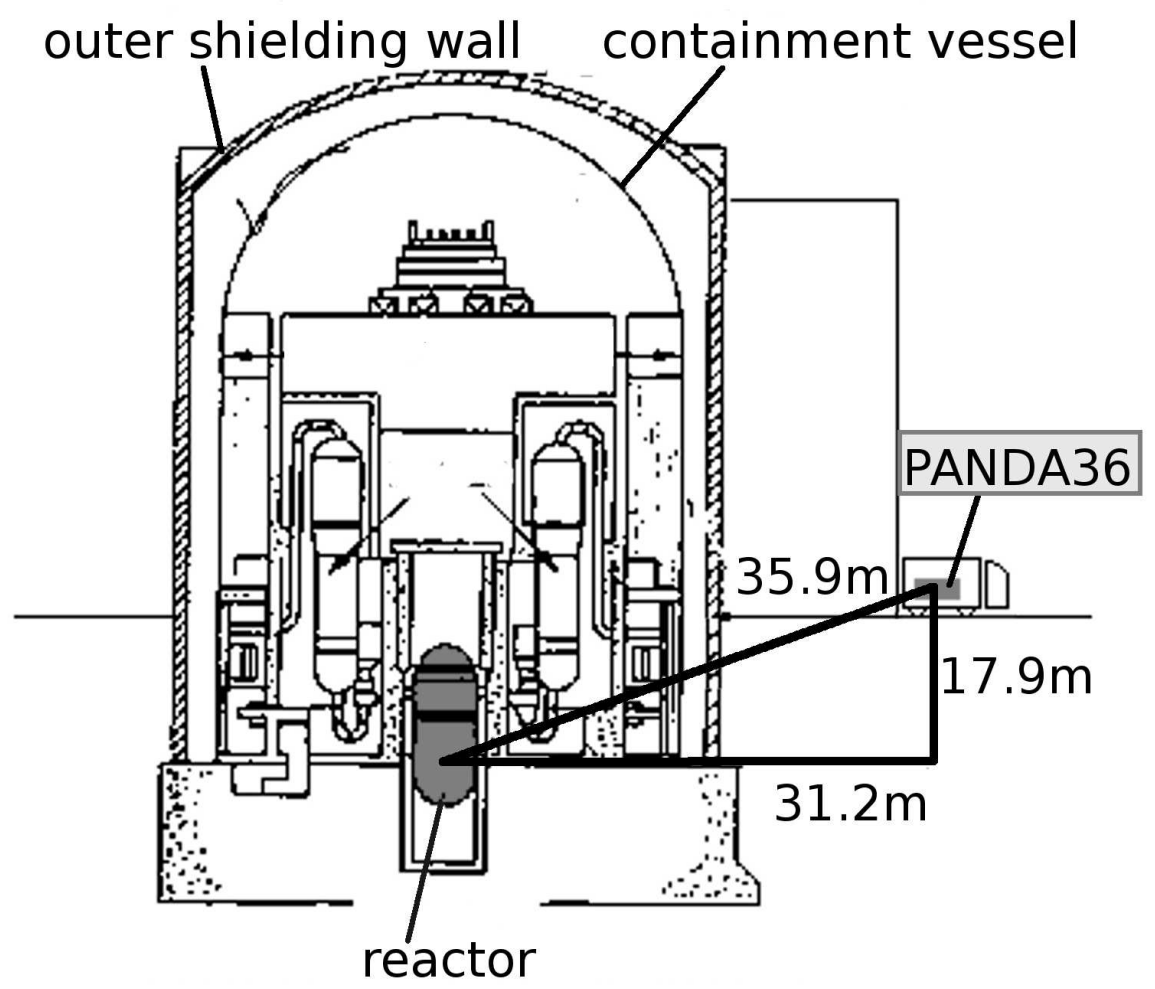

Figure 6: Location of PANDA36 at Ohi Power Station: the van was actually parked in parallel with the building wall. 
Table 2: Antineutrino and fast-neutron event selection criteria: Double underlines denote the difference between selection 1 and selection 2 .

\begin{tabular}{|c|c|c|}
\hline & selection 1 & selection 2 \\
\hline software trigger: & $\begin{array}{l}\text { At least two modules in } \\
\text { inner } 16 \text { modules deposite } \\
\text { energy of } 150 \mathrm{keV} \text { or more. }\end{array}$ & $\begin{array}{l}\text { At least two modules in } \\
\text { inner } 16 \text { modules deposite } \\
\text { energy of } 150 \mathrm{keV} \text { or more. }\end{array}$ \\
\hline prompt: & $\begin{array}{c}3 \mathrm{MeV} \leq E_{\text {total }} \leq 6 \mathrm{MeV} \\
\underline{\underline{E_{2 \mathrm{nd}}} \leq 520 \mathrm{keV}}\end{array}$ & $\begin{array}{c}3 \mathrm{MeV} \leq E_{\text {total }} \leq 6 \mathrm{MeV} \\
\underline{\underline{E_{2 \mathrm{nd}} \geq 700 \mathrm{keV}}}\end{array}$ \\
\hline delayed: & $\begin{aligned} & 3 \mathrm{MeV} \leq E_{\text {total }} \leq 8 \mathrm{MeV} \\
& \frac{E_{3 \mathrm{rd}}}{E_{\text {total }}} \geq \frac{E_{1 \mathrm{st}} / E_{\text {total }}-0.5}{5}\end{aligned}$ & $\begin{array}{c}3 \mathrm{MeV} \leq E_{\text {total }} \leq 8 \mathrm{MeV} \\
\frac{E_{3 \mathrm{rd}}}{E_{\text {total }}} \geq \frac{E_{1 \mathrm{st}} / E_{\text {total }}-0.5}{5}\end{array}$ \\
\hline time window: & $8 \mu \mathrm{s} \leq t \leq 150 \mu \mathrm{s}$ & $8 \mu \mathrm{s} \leq t \leq 50 \mu \mathrm{s}$ \\
\hline fiducial cut: & $\begin{array}{c}\text { The highest energy deposit } \\
\text { is in inner } 16 \text { modules }\end{array}$ & $\begin{array}{l}\text { The highest energy deposit } \\
\text { is in inner } 16 \text { modules }\end{array}$ \\
\hline muon veto: & $\begin{array}{c}\text { There is no event with } \\
E_{\text {total }}>8 \mathrm{MeV} \text { within } \\
250 \mu \text { s before the delayed } \\
\text { event. }\end{array}$ & $\begin{array}{c}\text { There is no event with } \\
E_{\text {total }}>8 \mathrm{MeV} \text { within } \\
250 \mu \text { s before the delayed } \\
\text { event. }\end{array}$ \\
\hline
\end{tabular}


ground events could not be discriminated from antineutrino events by selection 1. Therefore, we introduced selection 2 which is sensitive to fast neutron events.

It should be noted that other background, mainly uncorrelated events, cannot be fully eliminated by either of two selections because of high background rate at the surface. Therefore, $N_{\mathrm{s} 1}$ and $N_{\mathrm{s} 2}$, the number of events by selections 1 and 2 , respectively can be written as

$$
\begin{aligned}
& N_{\mathrm{s} 1}=\epsilon_{\nu, \mathrm{s} 1} N_{\nu}+\epsilon_{n, \mathrm{~s} 1} N_{n}+\epsilon_{\mathrm{B}, \mathrm{s} 1} N_{\mathrm{B}}, \\
& N_{\mathrm{s} 2}=\epsilon_{\nu, \mathrm{s} 2} N_{\nu}+\epsilon_{n, \mathrm{~s} 2} N_{n}+\epsilon_{\mathrm{B}, \mathrm{s} 2} N_{\mathrm{B}}
\end{aligned}
$$

where $N_{\nu}, N_{n}$ and $N_{\mathrm{B}}$ are numbers of antineutrino events, fast neutron events and other background events occurred in the detector, respectively. Coefficients $\epsilon_{\nu, \mathrm{s} 1}, \epsilon_{n, \mathrm{~s} 1}, \epsilon_{\mathrm{B}, \mathrm{s} 1}, \epsilon_{\nu, \mathrm{s} 2}, \epsilon_{n, \mathrm{~s} 2}$, and $\epsilon_{\mathrm{B}, \mathrm{s} 2}$ are the detection efficiencies of selections 1 and 2 for antineutrinos, fast neutrons and other background events.

From $N_{\mathrm{s} 1}$ we would like to evaluate the number of fast-neutron-free events $N_{\nu \mathrm{B}}$ by

$$
\begin{aligned}
N_{\nu \mathrm{B}} & \equiv N_{\mathrm{s} 1}-\frac{\epsilon_{n, \mathrm{~s} 1}}{\epsilon_{n, \mathrm{~s} 2}} N_{\mathrm{s} 2} \\
& =\left(\epsilon_{\nu, \mathrm{s} 1}-\epsilon_{\nu, \mathrm{s} 2} \frac{\epsilon_{n, \mathrm{~s} 1}}{\epsilon_{n, \mathrm{~s} 2}}\right) N_{\nu}+\left(\epsilon_{\mathrm{B}, \mathrm{s} 1}-\epsilon_{\mathrm{B}, \mathrm{s} 2} \frac{\epsilon_{n, s 1}}{\epsilon_{n, \mathrm{~s} 2}}\right) N_{\mathrm{B}} \\
& =\epsilon_{\nu, \mathrm{s} 1}\left(1-\frac{\epsilon_{\nu, \mathrm{s} 2} / \epsilon_{\nu, \mathrm{s} 1}}{\epsilon_{n, \mathrm{~s} 2} / \epsilon_{n, \mathrm{~s} 1}}\right) N_{\nu}+\left(\epsilon_{\mathrm{B}, \mathrm{s} 1}-\epsilon_{\mathrm{B}, \mathrm{s} 2} \frac{\epsilon_{n, s 1}}{\epsilon_{n, \mathrm{~s} 2}}\right) N_{\mathrm{B}}
\end{aligned}
$$

Consequently, $N_{\nu \mathrm{B}}$ should consist of antineutrino events and unnormalized uncorrelated background events, and is free from fast neutron events.

Detection efficiencies, $\epsilon_{\nu, \mathrm{s} 1}, \epsilon_{n, \mathrm{~s} 1}, \epsilon_{\nu, \mathrm{s} 2}$ and $\epsilon_{n, \mathrm{~s} 2}$, are estimated by Monte Carlo simulation using Geant4 toolkit [12]. Detection efficiency of selection 1 and the systematic errors are summarized in Tab. 3. A summary of the efficiency ratios, $\epsilon_{\nu, \mathrm{s} 2} / \epsilon_{\nu, \mathrm{s} 1}$, and $\epsilon_{n, \mathrm{~s} 2} / \epsilon_{n, \mathrm{~s} 1}$, and their systematic errors are shown in Tab. 4

The selection criteria of selections 1 and 2 are optimized as follows. First of all, a software trigger was applied to the data for the both selections before the analysis. Because the hardware thresholds are not necessarily the same for all 
Table 3: Summary of the detection efficiency and the systematic errors of selection 1: The detection efficiency of the delayed events is estimated for the simulated events which satisfied the prompt event selection. The detection efficiency of the time window is estimated for the simulated events which satisfied the prompt and delayed event selection. The relative error consists of the uncertainties in the simulation models "relative error(model)" and the PMT gain factors "relative error(gain)".

\begin{tabular}{ccccc}
\hline \hline & & efficiency & $\begin{array}{c}\text { relative } \\
\text { error(model) }\end{array}$ & $\begin{array}{c}\text { relative } \\
\text { error(gain) }\end{array}$ \\
\hline prompt & trigger & $28.6 \%$ & $12.1 \%^{(1)}$ & - \\
& $E_{\text {total }}$ cut & $44.2 \%$ & $10.5 \%$ & - \\
& $E_{\text {2nd }}$ cut & $82.2 \%$ & $12.1 \%(1)$ & - \\
& fiducial cut & $93.5 \%$ & $5.0 \%$ & - \\
& total & $9.7 \%$ & $16.8 \%$ & $3.4 \%$ \\
\hline delayed & trigger & $48.8 \%$ & $19.4 \%^{(2)}$ & - \\
& $E_{\text {total }}$ cut & $79.1 \%$ & $19.4 \%^{(2)}$ & - \\
& $E_{3 \mathrm{rd}}$ cut & $91.9 \%$ & $19.4 \%^{(2)}$ & - \\
& total & $35.5 \%$ & $19.4 \%$ & - \\
\hline time window & & $91.2 \%$ & $14.3 \%$ & $\pm 29.6 \%$ \\
\hline Total & $\epsilon_{\nu, \mathrm{s} 1}=$ & $3.15 \%$ & & \\
\hline
\end{tabular}

(1) The uncertainties in the software trigger efficiency and the prompt $E_{2 n d}$ selection are estimated as a whole.

(2) The uncertainties in the three criteria for the delayed events are estimated as a whole. 
Table 4: Summary of the efficiency ratios and the systematic errors

\begin{tabular}{clcc}
\hline \hline & & antineutrino $\left(\epsilon_{\nu, \mathrm{s} 2} / \epsilon_{\nu, \mathrm{s} 1}\right)$ & fast neutron $\left(\epsilon_{\mathrm{n}, \mathrm{s} 2} / \epsilon_{\mathrm{n}, \mathrm{s} 1}\right)$ \\
\hline relative error: & $E_{\text {2nd }}$ selection(model) & $27 \%$ & $14.4 \%$ \\
relative error: & $E_{2 \text { nd }}$ selection(gain) & $1.0 \%$ & $0.4 \%$ \\
relative error: & software trigger & - & $33.3 \%$ \\
relative error: & time window(model) & $1.1 \%$ & $1.1 \%$ \\
\multicolumn{2}{c}{ total relative error } & $27 \%$ & $36.3 \%$ \\
\hline & value & $0.086 \pm 0.023$ & $1.86 \pm 0.68$
\end{tabular}

the PMT's, it is required to apply the software trigger with a common threshold to estimate the appropriate trigger efficiency.

In the next step, we selected the prompt events by requiring the total energy $E_{\text {total }}$ to be in the range between 3 and $6 \mathrm{MeV}$ to reduce the environmental gamma-ray background. We expect that a prompt event consists of one positron and two annihilation gamma rays. In many cases, $E_{1 \text { st }}$ corresponds to the ionization loss of the positron and $E_{2 n d}$ corresponds to the Compton scattering of one of the annihilation gamma rays. Here, $E_{1 \mathrm{st}}$ and $E_{2 n d}$ are the highest and the second highest energy deposits among all the modules. $E_{3 \mathrm{rd}}$ is also similarly defined as the third highest deposit energy. To include the energy of $511 \mathrm{keV}$ of the annihilation gamma ray, $E_{2 \text { nd }}$ was required to be less than 520 $\mathrm{keV}$ for selection 1, and to be greater than $700 \mathrm{keV}$ for selection 2 to exclude positrons. The double-neutron correlated background events were also efficiently eliminated by the $E_{2 n d}$ cut of selection 1 . It is because the prompt event of the double-neutron correlated background is composed of high energy gamma ray cascade.

The delayed event is characterized by two or more gamma rays as a gamma ray cascade with a total energy of $7.9 \mathrm{MeV}$ emitted by ${ }^{157} \mathrm{Gd}$ and $8.5 \mathrm{MeV}$ emitted by ${ }^{155} \mathrm{Gd}$ following the thermal neutron captures common to both the selections. $E_{\text {total }}$ is, therefore, required to be in the range between 3 and 8 $\mathrm{MeV}$ for the selection of delayed events. It is rare for the energy deposit to 
be localized in one module because two or more gamma rays are emitted at a time in the delayed event. So, we expect that $E_{1 \mathrm{st}} / E_{\text {total }}$ is not much larger than $E_{2 \text { nd }} / E_{\text {total }}$ nor $E_{3 \mathrm{rd}} / E_{\text {total }}$. Fig. 7 shows scatter plots of $E_{3 \mathrm{rd}} / E_{\text {total }}$ vs $E_{1 \text { st }} / E_{\text {total }}$ of the simulation(above) and the observed data(below). As expected, the events of the observed data are concentrated on $E_{1 \text { st }} / E_{\text {total }} \sim 1$ because they are dominated by gamma ray background events, and the events of the simulation are scattered. We accordingly required

$$
\frac{E_{3 \mathrm{rd}}}{E_{\text {total }}} \geq \frac{E_{1 \mathrm{st}} / E_{\text {total }}-0.5}{5}
$$

in both selection 1 and selection 2 .

We paired a prompt-like event with the following delayed-like event when the delay time was within a predefined time window.

Due to the conversion time of the QDC(about $7.5 \mu \mathrm{s})$, the minimum threshold for the time window was set at $8 \mu \mathrm{s}$. The distributions of the prompt-delayed intervals by selection 1(top) and selection 2(bottom) are shown in Fig. 8

The dashed lines show the observed data and the solid lines show the simulation of neutrino events(top) and fast neutron correlated events(bottom). The simulation curves for both the selections are exponentially decreasing with time because the event pair is correlated. On the other hand, the data curves are decreasing more gradually. It is most probably due to accidental coincidence events pairs, which distribute constantly over the time.

Therefore, the shorter the time window is, the higher the fraction of the correlated events is selected. But setting shorter time window leads to reduction in detection efficiency. We set the time windows as

$$
8 \mu \mathrm{s} \leq t \leq 150 \mu \mathrm{s} \quad(\text { selection } 1)
$$

so as to get high efficiency for the low-rate antineutrino events, and

$$
8 \mu \mathrm{s} \leq t \leq 50 \mu \mathrm{s} \quad(\text { selection } 2)
$$

to get high-purity fast neutron events sample of sufficient rate even with low efficiency. 

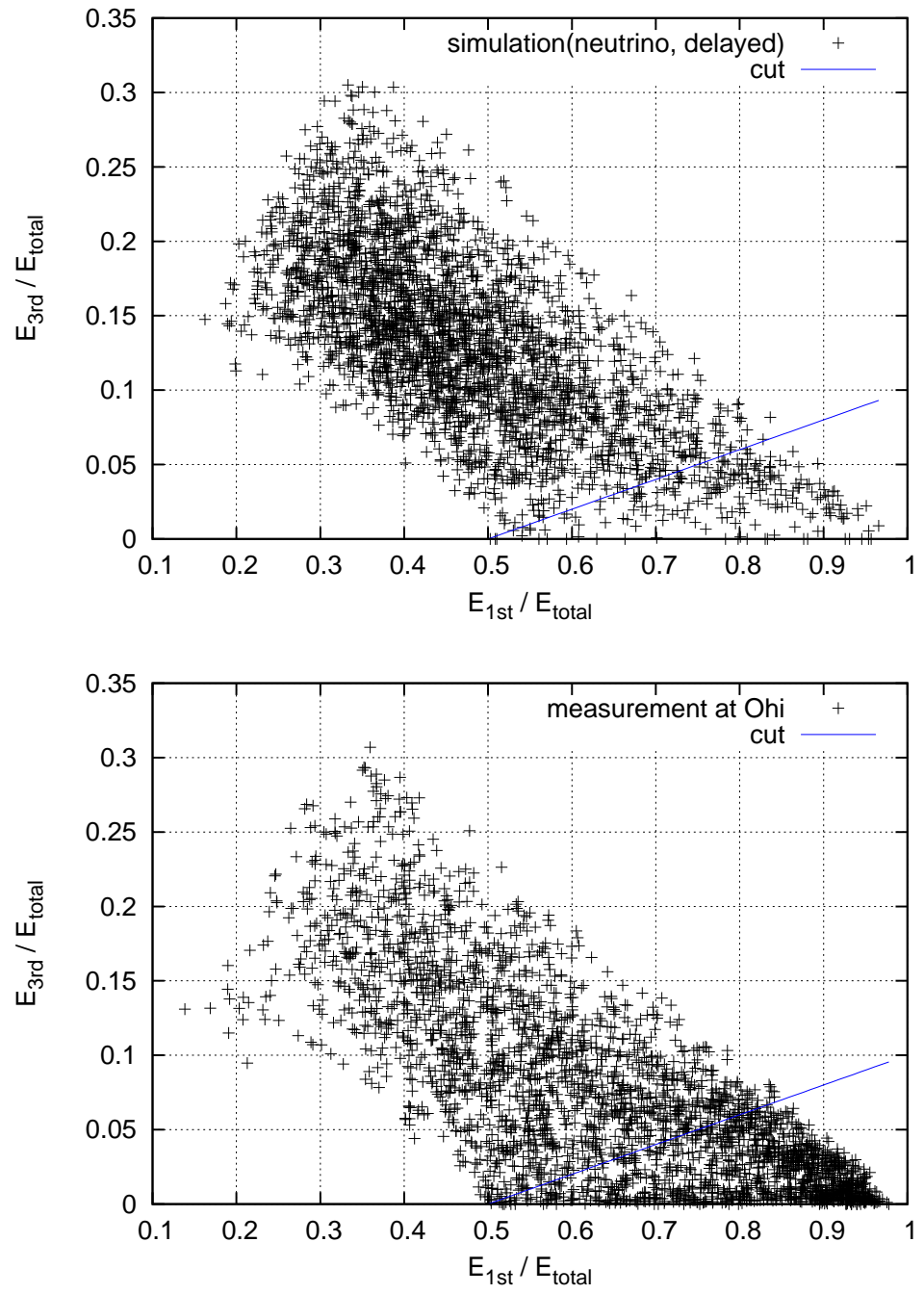

Figure 7: The above scatter plot shows the simulation and the below plot shows the observed data. The line shows the selection cut for delayed events $\left(\frac{E_{3 \mathrm{rd}}}{E_{\mathrm{total}}} \geq \frac{E_{1 \mathrm{st}} / E_{\mathrm{total}}-0.5}{5}\right)$. 

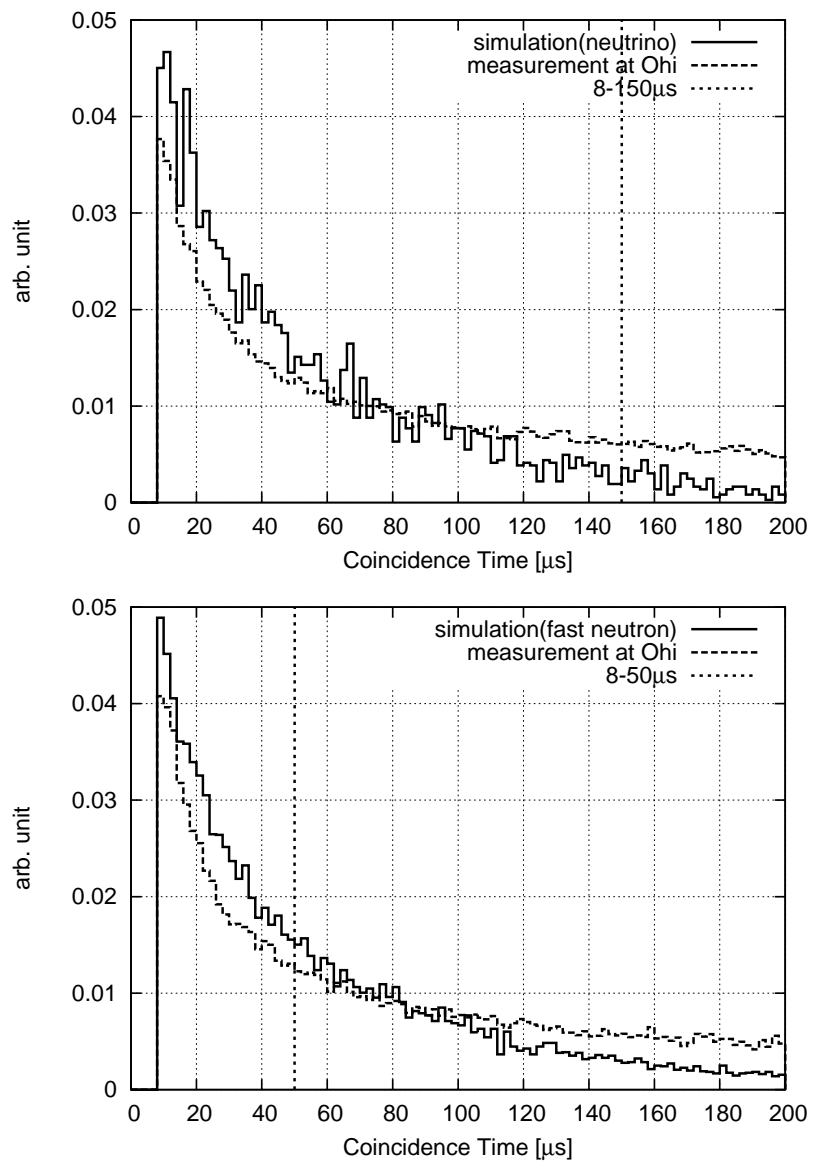

Figure 8: Distribution of the prompt-delayed interval: The prompt events satisfied the software trigger criterion, $E_{\text {total }}$ selection and $E_{2 \text { nd }}$ selection. The delayed events satisfied the software trigger criterion, $E_{\text {total }}$ selection and $E_{3 \mathrm{rd}} / E_{\text {total }}$ selection. (top) The solid and dashed lines show the simulated(antineutrino) and observed distributions which are selected by selection 1. (bottom) The solid and dashed lines show the simulated(fast neutron) and observed distributions which are selected by selection 2. We set the time windows as $8 \mu \mathrm{s} \leq t \leq 150 \mu \mathrm{s}($ selection 1$)$ and $8 \mu \mathrm{s} \leq t \leq 50 \mu \mathrm{s}($ selection 2$)$. 
Next we focus on the position of the highest energy deposit in the prompt event. Because $E_{1 \mathrm{st}}$ of the prompt event is supposed to correspond to the ionization loss of the positron, the position of the highest energy deposit is uniformly distributed in 36 modules. On the other hand, the prompt $E_{1 \mathrm{st}}$ of the correlated background by the fast neutron is supposed to correspond to the proton recoil. Because neutron interaction with a hydrogen nucleus at the neutron energy of $10 \mathrm{MeV}$ has a cross section of about 1 barn, neutrons have the mean free path of about $15 \mathrm{~cm}$ in the plastic scintillator. Therefore, the highest energy deposit tends to occur in outer 20 modules. However, it should be noted that because of the correlation with the software trigger criterion, both the distributions already concentrate on inside 16 modules. We cut the events whose prompt $E_{1 \mathrm{st}}$ module are located in outer 20 modules as a fiducial cut.

We introduce a muon veto cut by software. Cosmic ray muons can produce fast neutrons which bring on correlated background. We assume the event with $E_{\text {total }}$ of more than $8 \mathrm{MeV}$ is a muon candidate and rejected any prompt-delayed event pairs in which a muon candidate event occurred within $250 \mu$ s before the delayed event .

The detection efficiency of selection 1(Tab. 2) was calculated using the simulation toolkit Geant4. The systematic uncertainty of the efficiency was estimated as follows. We assumed that the systematic uncertainty consists of two different mechanisms, uncertainties in the simulation models and uncertainties in the PMT gain factors. To estimate the uncertainties in the simulation models, dedicated experiments with radioactive sources were carried out and the detection rates were compared between the observation and the simulation. To estimate the uncertainty in the prompt software trigger efficiency and the prompt $E_{2 \text { nd }}$ selection, we carried out an experiment with ${ }^{22} \mathrm{Na}$ positron- and gamma ray source. And to estimate the uncertainty in the selection criteria for delayed events, we carried out an experiment with ${ }^{252} \mathrm{Cf}$ neutron source. Because it is difficult to verify the prompt $E_{\text {total }}$ selection by experiments using radioactive sources, we calculated its uncertainty using the simulation result and the estimated energy resolution. To estimate the uncertainty of the detection efficiency 
attributed to the PMT gain variation, we also used the simulation result, but not experimental data.

The summary of the detection efficiency and the systematic errors is shown in Tab. 3. The detection efficiencies of the prompt event selection, the delayed event selection and the time window are affected by the position of the inverse beta decay. There are, therefore, correlations among them. The relative error of $29.6 \%$ and the detection efficiency of $(3.15 \pm 0.93) \%$ were estimated.

We need to estimate the efficiency ratios $\epsilon_{\nu, \mathrm{s} 2} / \epsilon_{\nu, \mathrm{s} 1}$ and $\epsilon_{n, \mathrm{~s} 2} / \epsilon_{n, \mathrm{~s} 1}$ to calculate $N_{\nu \mathrm{B}}$ in Eq.(7) and to evaluate the theoretical expectation by Eq.(9).

The ratios of the detection efficiencies were calculated using the simulation. The differences between selection 1 and selection 2 are the prompt $E_{2 \text { nd }}$ selection and the coincidence time window. Contributions of the other common selection criteria cancel out each other. Both uncertainties in the simulation models and the PMT gain factors contribute to the uncertainty in the prompt $E_{2 n d}$ selection. On the other hand, only the uncertainties in the simulation models contribute to the uncertainty of the coincidence time window cut. To estimate the uncertainties in the simulation models, we compared the energy spectra and the coincidence time distributions between the simulation and the observed data.

The expected antineutrino event rate was thus estimated by the factor of the first term of Eq.99). According to a report released by the Kansai Electric Power Co., Inc., the reactor generated the thermal power of $3.4 \pm 0.1 \mathrm{GW}$. The systematic error of the thermal power is not reported, so the very conservative value was used for this estimation. We assumed in this estimation that the fission fuel fraction is the same as the SONGS experiment[6]. It is simply assumed that all the fuel is concentrated at the center of the reactor core as it is a sufficient approximation [14] for the present experiment. The expected antineutrino detection rate by PANDA36(target mass: $360 \pm 18 \mathrm{~kg}$ ) is $17.3 \pm$ 6.2 events/day. 


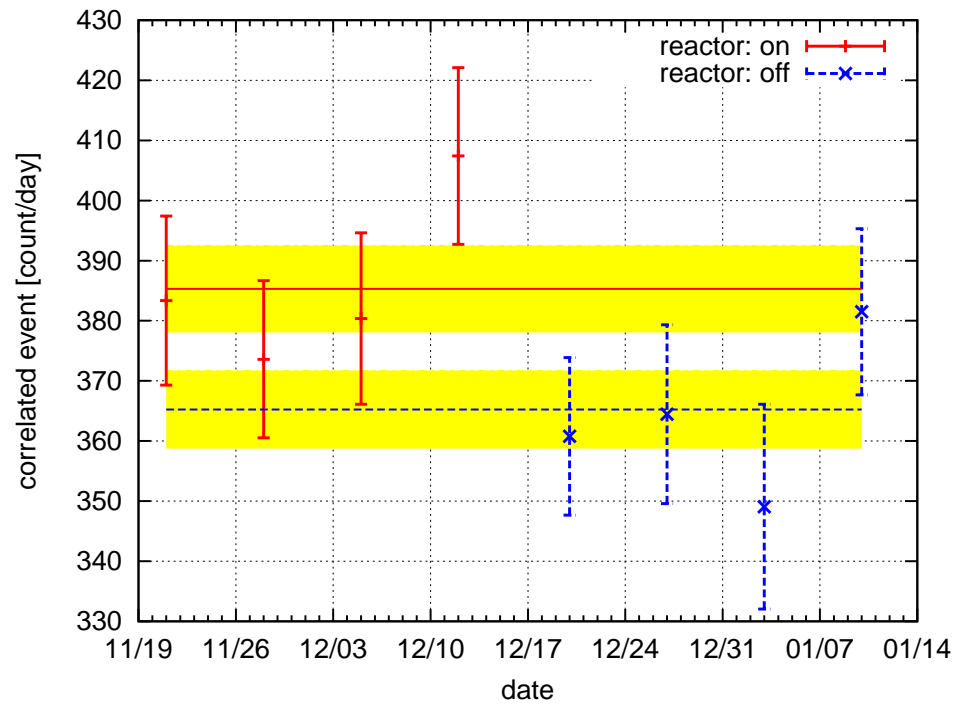

Figure 9: Seven day average daily event rates $N_{\nu \mathrm{B}}$

\section{Result}

We calculated the daily rates $N_{\nu \mathrm{B}}$ by Eq.(17) averaged for seven days and plotted them in Fig. 9. The reactor went shutdown on 16th December. The crosses and the stars show the data during the reactor ON and OFF period, respectively. The errors shown are statistical only because the systematic error of $\epsilon_{n, \mathrm{~s} 1} / \epsilon_{n, \mathrm{~s} 2}$ is attributed to the overall normalization of $N_{\nu \mathrm{B}}$. The horizontal bands represent averages and error interval of $N_{\nu \mathrm{B}}$ for the reactor ON period and OFF period.

The reactor ON/OFF difference of $N_{\nu \mathrm{B}}$ was evaluated to be $21.8 \pm 11.4$ events/day. The result is consistent with the predicted event rate of $17.3 \pm 6.2$ events/day.

We assumed the correlated background consists of only fast neutron events by now. We discuss other candidates of long-lived cosmic ray activation products in the following.

Beta decays of ${ }^{9} \mathrm{Li}$ and ${ }^{8} \mathrm{He}$ produced by cosmic muons could also cause 
correlated background. Decay rates of ${ }^{9} \mathrm{Li}$ and ${ }^{8} \mathrm{He}$ are estimated to be less than $4 \times 10^{-7} \mu^{-1} \mathrm{~g}^{-1} \mathrm{~cm}^{2}$ and $4 \times 10^{-8} \mu^{-1} \mathrm{~g}^{-1} \mathrm{~cm}^{2}$, respectively [13]. Assuming the vertical muon intensity of $1.0 \times 10^{-2} \mathrm{~cm}^{-2} \mathrm{sr}^{-1} \mathrm{~s}^{-1}$ and angular dependence of $\cos ^{2} \theta$, production rates of the isotopes in PANDA36 $\left(3.6 \times 10^{5} \mathrm{~g}\right)$ were estimated to be

$$
\begin{aligned}
& { }^{9} \mathrm{Li}: \quad 3.0 \times 10^{-3} \mathrm{~s}^{-1} \sim 260 \text { day }^{-1}, \\
& { }^{8} \mathrm{He}: \quad 3.0 \times 10^{-4} \mathrm{~s}^{-1} \sim 26 \text { day }^{-1} .
\end{aligned}
$$

Because the detection efficiency of less than $0.5 \%$ was calculated of each beta decay by the simulation and no ON/OFF difference in the muon flux is expected, contribution to the final result is negligible.

Natural radioisotopes ${ }^{220} \mathrm{Rn},{ }^{222} \mathrm{Rn}$ and their daughters emit $\alpha$ radiation with the energy range of 4-9 $\mathrm{MeV}(1-2 \mathrm{MeV}$ electron equivalent). If these isotopes permeate into the plastic scintillator, they might cause $(\alpha, \mathrm{n})$ reactions. Such events could also be observed as the correlated background by the delayed coincidence between the $\alpha$ ionization and the following neutron capture. However, the abundance of the radon near the detector in the open air is expected to be much less than under the ground, and the plastic scintillator is less permeable by radon than liquid scintillator. In addition to that, no ON/OFF difference is expected in the event rate from radon and their daughters because the radon is not generated by the fission in the reactor. It should be noted that the prompt event of the $(\alpha, \mathrm{n})$ reaction is rejected by the $E_{\text {total }}$ cut of selection 1 and selection 2 .

\section{Prospect}

We plan to build PANDA100, an antineutrino detector with $10 \times 10$ modules, as our ultimate goal by upgrading PANDA36, possibly with an intermediate prototype PANDA64 with $8 \times 8$ modules. They are expected to have higher detection efficiency than PANDA36 in addition to a larger target mass because the escape of the cascade gamma rays following neutron capture is suppressed with the larger volume. 
Antineutrino detection efficiency of PANDA100 is estimated to be $9.24 \%$ using simulation by applying a selection similar to selection 1. If PANDA100 were deployed at the same position as PANDA36 at Ohi Power Station, the selected antineutrino event rate would be $\sim 147$ events/day. Background rates of PANDA100 can also be estimated assuming the same background fast neutron flux as PANDA36. PANDA100 is thus expected to be able to detect the change of the reactor status by more than $5 \sigma$ in a week aboveground and to achieve the IAEA's medium term goals.

In order to make more precise measurement of the antineutrino flux, we have to reject more background events. As discussed in the former sections, the main source of the background is the "proton recoil - neutron capture" events by fast neutrons. One of the solutions to the background rejection is to shield the detector from fast neutrons with water tanks or polyethylene blocks. Our detector has the ability to discriminate between the prompt events and the delayed events. So if fast neutrons could be thermalized before reaching the detector, we would be able to reject the background events of fast neutrons.

\section{Conclusion}

We developed the prototype of the reactor antineutrino detector as a new safeguards tool and demonstrated the almost unmanned field operation at the reactor site for two months. We observed the difference of the reactor antineutrino flux with the reactor ON and OFF even with small 360-kg prototype detector above the ground in the vicinity of a commercial reactor of a power plant for the first time.

Our detection efficiency of the inverse beta decay is $3.15 \pm 0.93 \%$. We installed the detector at $35.9 \pm 0.1$ meters away from the $3.4 \pm 0.1 \mathrm{GW}_{\text {th }}$ reactor core. The difference of the antineutrino event rate between the reactor ON period and the reactor OFF period is $21.8 \pm 11.4$ events/day. The predicted difference is $17.3 \pm 6.2$ events/day.

Assuming the fast neutron flux measured by the PANDA36 experiment, the 
ultimate 100-module detector, PANDA100, is expected to be able to detect the change of the reactor status by more than $5 \sigma$ in a week aboveground and to achieve the IAEA medium term goals.

\section{Acknowledgements}

The authors thank the Kansai Electric Power Co., Inc. for its cooperation for our experiment on site of Ohi Power Station. They are also grateful to Professor Yoichi Fujiie for his support for this research project. The authors wish to acknowledge useful discussions with professor Yoichiro Shimazu. This research was partially supported by the Japanese Ministry of Education, Science, Sports and Culture, Grant-in-Aid for COE Research, Grant-in-Aid for Scientific Research (B), and Grant-in-Aid for JSPS Fellows and also by the Mitsubishi Foundation.

\section{References}

[1] C. L. Cowan, Jr., F. Reines, F. B. Harrison, H. W. Kruse and A. D. McGuire, Science 124 (1956)103.

[2] P. Vogel, J. Engel, Phys. Rev. D 39 (1989) 3378.

[3] T. A. Mueller, D. Lhuillier, M. Fallot, A. Letourneau, S. Cormon, M. Fechner, L. Giot, T. Lasserre, J. Martino, G. Mention, A. Porta, F. Yermia, Phys. Rev. C 83 (2011) 054615.

[4] P. Huber, Phys. Rev. C 84 (2011) 024617.

[5] IAEA Headquarters. Final Report: Focused Workshop on Antineutrino Detection for safeguards Applications, Oct 2008.

[6] A. Bernstein, Y. Wang, G. Gratta, T. West, J. Appl. Phys. 91 (2002) 4672, N. S. Bowden. J. Phys. Conf. Ser., 136 (2008) 022008, A. Bernstein, N. S. Bowden, A. Misner, T. Palmer, J. Appl. Phys. 103 (2008) 074905, N. S. 
Bowden, A. Bernstein, S. Dazeley, R. Svoboda, A. Misner, T. Palmer, J. Appl. Phys. 105 (2009) 064902.

[7] Y. V. Klimov, V. I. Kopeikin, L. A. Mikaelyan, K. V. Ozerov, V. V. Sinev, Atomic Energy 76 (1994) 123.

[8] H. Furuta et al., Nucl. Instrum. Meth. A662 (2012) 90.

[9] V. Belov et al., J. Instrumentation 8 (2013) P05018, I. Alekseev et al., arXiv:1305.3350[physics.ins-det], M. Danilov (DANSS Collaboration), arXiv:1311.2777[physics.ins-det].

[10] A. Porta et al. ( Nucifer coll.), Journal of Physics: Conf. Ser. 203 (2010) 012092, A.S. Cucoanes (Nucifer Collaboration), J. Phys. Conf. Ser. 375 (2012) 042063.

[11] Yasuhiro Kuroda, Shugo Oguri, Yo Kato, Ryoko Nakata, Yoshizumi Inoue, Chikara Ito, Makoto Minowa, Nucl. Instrum. Meth. A690 (2012) 41.

[12] S. Agostinelli, et al., Nucl. Instrum. Meth. A506 (2003) 250.

[13] S. Abe et al. (KamLAND Collaboration), Phys. Rev. C81 (2010) 025807.

[14] Yoichiro Shimazu, private communication. 